\title{
Refractory migraine: the role of the physician in assessment and treatment of a problematic disease
}

\author{
Bruno Colombo $\cdot$ Dacia Dalla Libera • \\ Gloria Dalla Costa $\cdot$ Giancarlo Comi
}

(C) Springer-Verlag Italia 2013

\begin{abstract}
Patients affected by chronic forms of headache are often very difficult to treat. Refractory patients are so defined when adequate trials of specific drugs (for acute or prophylactic treatment) failed both to reduce the burden of disease and to improve headache-related quality of life. An escalating approach is suggested to test different kinds of therapies. All comorbid factors should be addressed. More invasive modalities (such as neurostimulation) or promising approaches such as repetitive transcranial magnetic stimulation (rTMS) could be a future major step as third line therapies.
\end{abstract}

Keywords Chronic headache - Refractory migraine · Intractable headache $\cdot$ Escalating therapy

\section{Introduction}

Chronic forms of primary headaches, characterized by daily or almost daily attacks, are the most disabling forms of headache and have a dramatic impact on patients' ability to perform daily activities [1].

Furthermore, these forms make up a large percentage of new visits to tertiary care centers [2].

Chronic migraine $(\mathrm{CM})$ is a complication of relapsing migraine with $2.5 \%$ of the patients progressing yearly from an episodic to a chronic form.

B. Colombo $(\varangle)$ - D. Dalla Libera · G. Dalla Costa · G. Comi Department of Neurology, Headache Center and Institute of Experimental Neurology, IRCCS San Raffaele Hospital, Vita-Salute University, Via Olgettina 48, 20100 Milan, Italy e-mail: colombo.bruno@hsr.it
Nevertheless, only one-third of CM patients are using pharmacological prophylaxis mainly because of the low rate of correct diagnosis $(20 \%)$ [3].

Many patients suffering from chronic headache are either resistant or intolerant to current pharmacological regimens and meet the definition of "refractoriness".

Refractory migraine is a puzzling problem in clinical practice. Patients with this diagnosis are a challenge to the treating physician, due to the real difficulty in finding an alternative and adequate therapeutic plan. Considering previous uneffective attempts of treatment, patients with refractory migraine have to be considered a subset of complex and very problematic headache sufferers.

\section{Refractory migraine: a problem to solve}

Physician has the necessity to clearly define who is the real "refractory" patient.

A correct definition does serve as a starting point (a sort of common language for the community of headache specialists) to classify and identify this particular subset of patients.

Up to now, the definition of refractory migraine is still debatable, considering lack of response to pharmacologic treatments. In fact, patients suffer from a persistent headache that is very difficult to treat or fails to respond to common pharmacological trials. The term "intractable" headache could overlap the meaning of "refractory" if we consider the difficulty in relieving pain with standard options.

Albeit in literature few formal and operational definitions for refractory or intractable headache have been proposed, none has been accepted and incorporated into the 
International Classification of Headache Disorders (ICHD) $[4,5]$.

In practical terms, one could separate patient's refractoriness to preventive treatments from refractoriness to acute treatments: in any case, the final goal for the treating physician is to improve quality of life, ameliorate social interactions and reduce disability.

In acute treatment, the term "refractory" could be attributed to patients who fail to respond to standard treatments, or intolerant to the specific drugs.

To define refractory migraine based on response to acute treatments, a graded classification (class I to III) was recently proposed [6]. This classification is based on stratification of "intractability" defined as pathological severity. The incremental failure to different classes of drugs is the basic point of this classification. Class $\mathrm{I}$ is defined as the mildest scenario and includes patients who failed to achieve a significant response to two different classes of non-steroidal anti-inflammatory drugs (NSAIDs) or combination analgesics. Class II is a moderate condition and includes an inadequate response to NSAIDs along with triptans, antiemetics or ergot derivatives. Class III is the worst (most severe) situation and includes failure to all analgesics, oral or parenteral opioids, and corticosteroids.

The same authors considered the definition of refractory migraine based on response to preventive drugs.

A graded classification (class I to IV) was proposed. Class I is defined as failing a single trial of a proven preventive therapy or combination therapies. Class II is defined as failing two of these drugs. Class III is a severe condition including failure of an adequate trial of 3 preventive agents. Class IV is defined as the very severe form and includes patients who have failed aggressive infusions or detoxification protocols (when medication overuse coexists).

This proposed classification allows stratification of difficulty in treatment based on illness severity as mirrored by the continuous failure to an increased number of therapies.

In literature, the meaning of treatment failure is actually not specifically defined: the reduction in frequency and severity of attacks and the duration of treatment required for determining an absolute pharmacological failure is still not uniformed. In general, a $<50 \%$ reduction in frequency or severity of headache attacks is accepted [7]. Number of analgesics consumed, number of working days lost and headache-related disability are also important criteria to consider (Table 1).

The cause of treatment failure has to be determined, if possible. It is accepted that if therapy is basically inadequate (low dose, wrong class of medication, unrecognized exacerbating factors or incorrect duration of pharmacological test), the same failure is to be reassessed.
Another explanation for a treatment failure could be a bad treatment adherence, defined as the duration of time from initiation to discontinuation of therapy.

A universally accepted definition of refractory headache could help in determining the best management and pharmacological approach. In defining intractable headaches, other important variables such as psychiatric comorbidities, medical comorbidities (i.e., fibromyalgia or chronic fatigue) and level of disability have to be considered. The approach could change depending on age, number of years of headache and exacerbating factors (i.e., hormone factors) [8].

The identification and the correct treatment of all clinically important comorbidities are mandatory before stating a patient's refractoriness.

The definition of different levels of intractability is a good tool to address patients through defined treatment protocols in an "escalating" way, identifying who could utilize more aggressive therapies according to the severity of the disease in terms of reduction of quality of life.

Escalation could be defined as a therapeutic strategy based on a reasonable decision-making procedure in which drugs with the best risk-benefit ratio are the first preferred and, if needed, drugs or alternative approaches with increasing power or toxicity (but not necessarily more effective) are successively adopted (Table 2).

In the escalating protocol, particularly in prevention, the failure of a first treatment raises the opportunity to switch to an alternative drug of the same line (combination therapy) or to move to the next level (with more putative risk of adverse events).

Combination therapy has the potential advantage to target different aspects of the pain dysfunction, with potentially better results due to the synergistic effects of different treatments.

However, there is insufficient evidence to state definitely whether combination therapy is clearly superior to single therapy [7].

Considering third level therapies, central and peripheral neurostimulation are of growing interest for the treatment of refractory headache patients.

Patients who are completely drug resistant and with severe disability (i.e. patients who do not respond to all

Table 1 Towards a comprehensive refractory migraine definition

Failure of first and second line treatments in adequate doses (mono and polytherapy). 3-month treatment period is required to assess efficacy for each treatment. Failure is defined as no therapeutic effect or not tolerable side effects

Comorbidity optimally addressed

High degree of disability, poor quality of life (MIDAS scale)

Medication overuse correlation 
Table 2 Prophylactic therapies used for "escalating approach" (according to level of intractability)

\author{
First line \\ 1) Beta blockers \\ 2) Tricyclic antidepressants, SSRI (shown to be effective in \\ RCT's) \\ 3) Flunarizine or verapamil \\ 4) Pizotifen \\ Second line \\ 1) Sodium valproate \\ 2) Topiramate \\ 3) Gabapentin \\ Third line \\ 1) Combination therapy (one or two drugs of first line-evaluating \\ possible pharmacological interactions - or one drug of first line \\ and one drug of second line-evaluating possible \\ pharmacological interactions). Consider complementary \\ alternative compounds. \\ 2) Botox type-A \\ 3) Detoxification and steroids \\ 4) Neurostimulation therapies \\ Complementary alternative compounds \\ 1) Metabolic enhancers (vitamin B2 or CoQ10) \\ 2) Ginkgolide B \\ 3) Tanacetum parthenium
}

known medications) could be considered for neurostimulation procedures.

In selecting patients for neurostimulation, it is important to consider the complexity of the clinical picture. Analgesic overuse, psychiatric comorbidities, cardiovascular disease or hypertension, musculo-skeletal pain syndromes have to be characterized. Each single patient has to be evaluated by a multidisciplinary team, focusing on quality of life and burden of disease [9].

Small pivotal studies of occipital nerve stimulation in CM have shown encouraging results. A long-term followup is necessary to establish a significative efficacy of this procedure [10].

A novel and promising approach in $\mathrm{CM}$ is repetitive transcranial magnetic stimulation (rTMS, applied to prefrontal areas at $110 \%$ of motor threshold intensity and with a stimulation frequency of $20 \mathrm{~Hz}$ ).

Prefrontal cortex has a pivotal role in controlling pain modulatory pathways, particularly by driving endogenous pain-inhibitory circuits and is also involved in influencing the functionality of periaqueductal grey (PAG), anterior insula and amygdala (interconnectivity in the limbic system). Prefrontal cortex exerts a pain-reducing top-down activity, being hypoactive in CM.

rTMS may be able to modulate the activity of corticosubcortical and cortico-cortical pathways involved in pain control. In a pilot study, a significative reduction in headache days, migraine attacks and pain intensity was obtained together with improvement in quality of life in a group of chronic and refractory migraine patients [11].

These results are in agreement with a recent proposed dysfunctional model (the neurolimbic pain network model) of migraine. In this model, brainstem pain-modulating circuits have bidirectional connections with the limbic system (comprising prefrontal cortex) and tonically influence migraine expression. As episodic migraine progresses to $\mathrm{CM}$ and refractory migraine, neurolimbic dysfunction often increases [12].

These studies may yield insight to enable physicians to offer their "refractory" patients new specific therapies and, ultimately, greater pain relief.

\section{Concluding remarks}

The challenge of the future for refractory patients will be to find new therapeutical approaches specifically acting on neurolimbic pain networks, both to better modulate the functional connectivity between pain structures and to ameliorate or sustain the "limbic factors" such as mood, personality and emotion.

In this comprehensive view of refractory headache, an optimal mutual doctor-patient relationship yields the most positive treatment outcome. Compilation of headache diaries and calendar is suggested [13].

Change of lifestyle (sleep habits, exercise and diet) has to be discussed. Other non pharmacological treatments (including relaxation exercises and cognitive therapy) may serve to support wellbeing.

The flexibility about exploring treatment options and a cooperative attitude toward refractory patients is essential for the successful management of this problematic subgroup of headache sufferers.

Conflict of interest The author certifies that there is no actual or potential conflict of interest in relation to this article.

\section{References}

1. Evans RW (2013) A rational approach to the management of chronic migraine. Headache 53:168-176

2. Munakata J, Hazard E, Serrano D et al (2009) Economic burden of transformed migraine: results from the American Migraine Prevalence and Prevention (AMPP) Study. Headache 49:498-508

3. Bigal ME, Serrano D, Reed M et al (2008) Chronic migraine in the population: burden, diagnosis, and satisfaction with treatment. Neurology 71:559-566

4. Goadsby PJ, Schoenen J, Ferrari MD et al (2006) Towards a definition of intractable headache for use in clinical practice and trials. Cephalalgia 26:1168-1170

5. Schulman EA, Lake AE 3rd, Goadsby PJ et al (2008) Defining refractory migraine and refractory chronic migraine: proposed 
criteria from the Refractory Headache Special Interest Section of the American Headache Society. Headache 48:778-782

6. Silberstein SD, Dodick DW, Pearlman S (2010) Defining the pharmacologically intractable headache for clinical trials and clinical practice. Headache 50:1499-1506

7. D'Amico D, Leone M, Grazzi L et al (2008) When should "chronic migraine" patients be considered "refractory" to pharmacological prophylaxis? Neurol Sci 29(Suppl 1):S55-S58

8. Schulman E, McGeeney BE (2013) Current concepts in refractory migraine. Curr Treat Options Neurol 15:40-55

9. Proietti Cecchini A, Leone M, Manzoni GC et al (2012) Drugresistant chronic migraine: the Italian GON project. Neurol Sci 33(Suppl 1):S33-S35
10. Magis D, Jensen R, Schoenen J (2012) Neurostimulation therapies for primary headache disorders: present and future. Curr Opin Neurol 25:269-276

11. Dalla Libera D, Colombo B, Nuara A et al (2013) Repetitive transcranial magnetic stimulation (rTMS) as preventive treatment of chronic migraine: a safe approach with a promising effect. Paper presented at 64th AAN, on oral communication, San Diego, March 2013, pS59.005

12. Maizels M, Aurora S, Heinricher M (2012) Beyond neurovascular: migraine as a dysfunctional neurolimbic pain network. Headache. doi:10.1111/j.1526-4610.2012.02209.x

13. Nappi G, Jensen R, Nappi RE et al (2006) Diaries and calendars for migraine. A review. Cephalalgia 26:905-916 